\section{Potassium gymnemate and the sweet and bitter taste provoked electrically*}

\author{
ZORAN BUJAS and CARL PFAFFMANN \\ The Rockefeller University, New York, New York 10021
}

The sweetness or bitterness of taste solutions of saccharin and quinine may be blocked, while the sweetness or bitterness evoked by electrical stimulation of the tongue by cathodal polarization is unchanged. Electrical taste stimulation apparently bypasses the most peripheral process by which taste solutions elicit sweet and bitter and thus acts directly on the receptor and/or its afferent nerve terminals.

As is well known, potassium gymnemate, the extract of the plant Gymnema sylvestre, temporarily, but very markedly diminishes the sensitivity to sweet substances and, to a minor extent, also the sensitivity to bitter substances. This suppressive action seems to be common for all substances with these tastes, since potassium gymnemate inhibits, to the same degree, the sweet sensitivity to such chemically different substances as saccharin and sucrose (Warren \& Pfaffmann, 1959). It is also possible to provoke sweet and bitter tastes not only by taste solutions but by a continuous or iterative electrical stimulation of the tongue (v. Békésy, 1964). A continuous dc cathodal stimulus or iterative cathodal pulses of high frequency can elicit a complex taste quality, which the majority of Ss identify as bitter and/or sweet. Sometimes, especially when the cathodal stimulus is strong, some Ss also report a sour or salty taste, but usually these latter taste qualities rarely appear. Stimulation by "make" of dc anodal currents causes a predominantly sour taste. Since hypotheses as to the mechanism of the cathodal electric taste differ as between direct stimulation of taste afferent structures vs chemical action by electrolytic products, it seemed worthwhile to investigate the influence of potassium gymnemate on the sweet and bitter taste produced electrically.

Measurements of sensitivity for the "make" and "break" of cathodal current, for "make" of anodal current, and for the solutions of sodium saccharin and quinine hydrochloride were carried out. First, the thresholds for the above stimuli were determined. Then the extract of Gymnema sylvestre leaves (containing about $5 \%$ potassium gymnate) was applied to the

*This research was supported in part by a grant from the National Science Foundation, GB-4198, while Dr. Bujas was a Visiting Professor on the Inter-Academy Exchange Program between the U.S. National Academy of Sciences and the Council of the Academies of Yugoslavia. several times during $1 \mathrm{~min}$. Following this treatment and after rinsing the mouth with water, the thresholds for electrical and chemical stimuli were again determined.

Recognition threshold measurements were made by the ascending method of limits. The stimulation with electric current was continuous (dc) from controlled power source because the iterative stimulation did not produce the sweet and bitter tastes any more clearly than did the continuous stimulus. Nonpolarizable electrodes of chlorided silver wires in a solution of $.1 \%$ sodium chloride were employed. The surface of the active electrode consisted of a $50 \mathrm{~mm}^{2}$ agar plug in the end of a glass tube, which was placed and held by the $\mathbf{S}$ more or less on the same spot on the anterior tongue each time. A large indifferent electrode was provided by a bath for the fingers, giving surface area greater than $100 \mathrm{~cm}^{2}$. A resistance of $0.6-1.0$ megohm was inserted in series with the electrodes. The stimulus was turned on and off by a manual switch for a duration of $3 \mathrm{sec}$ after a ready signal. Stimuli were separated by an interval of 30 sec. The determination of thresholds before and following potassium gymnemate treatment required about $30-40 \mathrm{~min}$. Four Ss participated in the experiment, each in two sessions. For each $S$, the thresholds for the "make" of 20 series before and 20 series after the application of the extract, while the thresholds for the anodal current and for the cathodal "break" are the result of 10 series. In the normal condition, three Ss reacted to cathodal "make" stimulation with the bitter and/or sweet taste and very rarely with the sour, while one $S$ always reported a sweet-salt taste. For cathodal "break" or anodal "make," the predominant quality reported was sour. Anodal "break" was not studied.

A rough check of the sensitivity to saccharin and quinine solutions, at the beginning and at the end of the experiment, served only to determine anterior tongue surface by a brush of cathodal current are the mean value if the suppressive action of the gymnemate persisted during the interval required for determining the thresholds for electrical stimuli. This control showed that at 30 or $40 \mathrm{~min}$ following the application of the extract, the saccharin threshold was still found to be elevated by 50 -fold and the quinine threshold about 2.4-fold. The thresholds for electrical stimuli were reliable, and their magnitude and range were the same for all Ss. Both before and after treatment, the lowest threshold found was for the anodal "make" at 4 microA, the next higher for the cathodal "break" at 8 microA, and the highest for the "make" of cathodal current at 68 microA.

As expected, potassium gymnemate did not show any influence on the sensitivity to or the occurrence of the sour taste provoked by the anodal "make" or the cathodal "break." The average threshold values after the application of potassium gymnemate, expressed in the units of the threshold before treatment, were: for the anodal "make" current, $1.15(0.91,1.00$, $1.01,1.67)$, and for the cathodal "break," $0.95(0.75,0.96,1.15)$. The threshold for the cathodal "break" was determined only for three $S$ s because one $S$ responded inconsistently to the stimulus.

However, what was not foreseen was that the recognition threshold for bitter-sweet taste at the "make" of cathodal current did not change after the application of potassium gymnemate, the average threshold being $1.04(0.98,1.00,1.06,1.10)$, which is only $4 \%$ higher than before treatment. It is of interest that the spectrum of taste qualities reported also remained unchanged. Figure 1 shows the relative frequency of occurrence of different taste qualities

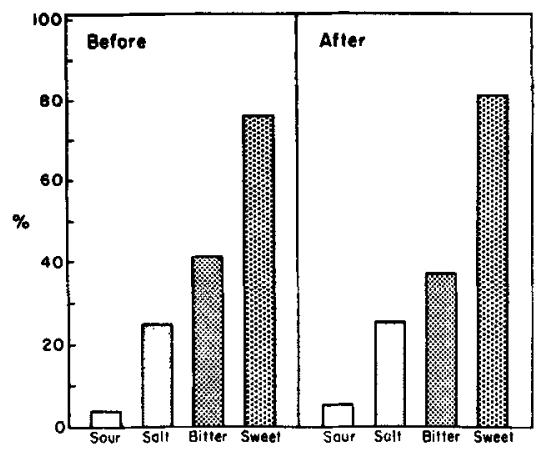

Fig. 1. Taste spectrum for the "make" of cathodal current before (a) and after (b) the application of $5 \%$ potassium gymnemate. Ordinate: frequencies of the occurrence of different taste qualities in percent. Total exceeds $100 \%$ because some qualities appeared in combination. 
produced by the "make" of cathodal current before and after the application of potassium gymnemate. The spectra represent the average values for four Ss based on $\mathbf{8 0}$ judgments. Potassium gymnemate, which so evidently depresses the sensitivity to sweet and bitter solutions, has no influence on these tastes when provoked electrically. The slight differences between the thresholds and between the frequencies of taste qualities before and after treatment are not statistically significant.

If we assume that gymnemic acid primarily blocks the receptor chemical site or chemical stimulation at the receptor cell membrane involved in sweet and bitter stimulation, then electrical stimulation seems to bypass this chemical step (Beidler, 1954). The stimulation of afferent structures by electrolytic breakdown processes, which then stimulate the afferent endings in the usual manner, as when chemicals are applied to the tongue surface, appears ruled out. One possibility is that the receptor cell is bypassed entirely and that stimulation occurs at the receptor cell-neural junction or nerve terminals. Whatever the details of this process, it is clear that sweet sensitivity to solutions depends on certain different intermediate steps than those invoked by electrical stimulation.

\section{REFERENCES}

BEIDLER, L. M. A theory of taste stimulation. Journal of General Physiology, 1954, 38, 133-139.

BEKESY, G. von. Sweetness produced electrically on the tongue and its relation to taste theories. Journal of Applied Physiology, 1964, 19, 1105-1113.

WARREN、R. M., \& PFAFFMANN, C. Suppression of sweet sensitivity by potassium gymnemate. Journal of Applied Physiology, 1959, 14, 40-42.

(Accepted for publication December 7, 1970.) 